\title{
Observations of simultaneous coronal loop shrinkage and expansion during the decay phase of a solar flare
}

\author{
J. I. Khan ${ }^{1}$, L. Fletcher ${ }^{1}$, and N. V. Nitta ${ }^{2}$ \\ 1 Department of Physics and Astronomy, University of Glasgow, Glasgow G12 8QQ, Scotland, UK \\ e-mail: jkhan@spd.aas.org \\ 2 Lockheed Martin Solar and Astrophysics Laboratory, 3251 Hanover Street, Palo Alto, CA 94304, USA \\ Received 29 November 2005 / Accepted 9 March 2006
}

ABSTRACT

\begin{abstract}
We report what we believe are the first direct and unambiguous observations of simultaneous coronal magnetic flux loop shrinkage and expansion during the decay phase of a solar flare. The retracting and expanding loops were observed nearly face-on (i.e., with the loop major axis approximately orthogonal to the line of sight) in emission in imaging data from the Yohkoh Soft X-ray Telescope (SXT). The retracting loop is observed to shrink with a speed of $118 \pm 66 \mathrm{~km} \mathrm{~s}^{-1}$. The faint outward moving loop-like feature occurred $\sim 200^{\prime \prime}$ above the shrinking loop during the time of the shrinking loop. We estimate the speed of the outward moving loop was $\sim 129 \pm$ $74 \mathrm{~km} \mathrm{~s}^{-1}$. We interpret the shrinking loop and simultaneous outward moving loop as direct evidence for reconnected magnetic field lines during a flare.
\end{abstract}

Key words. Sun: corona - Sun: flares - Sun: magnetic fields - Sun: X-rays, gamma rays - magnetohydrodynamics (MHD)

\section{Introduction}

Using images from the Yohkoh Soft X-ray Telescope (SXT) (Tsuneta et al. 1991) non-flaring coronal loops from solar active regions have been shown sometimes to exhibit shrinkage in the context of active region development (Wang et al. 1997). However, it is in the context of the energy release process in solar flares that coronal loop shrinkage (or retraction) is of particular interest. The energy release in flares is usually attributed to magnetic reconnection and from theoretical considerations and numerical simulations, newly reconnected magnetic field lines, in the form of closed magnetic loop structures anchored in lower atmospheric layers, should retract sunward after their formation (Priest \& Forbes 2000). Thus, coronal loop retraction is a natural consequence, and hence prediction, of some magnetic reconnection models, such as the models of Hirayama (1974) and Kopp \& Pneuman (1976). Moreover, based on the requirement that the volume integral of the magnetic energy density should decrease with time to release stored magnetic energy during a flare, Hudson (2000) conjectured that this necessarily required a shrinking or contraction of magnetic field lines, which he referred to as a magnetic implosion, independent of any reconnection scenario.

Indirect evidence for coronal magnetic loop shrinkage during solar flares has been inferred from some observations. For example, Švestka et al. (1987) inferred that if soft X-ray emitting loops observed during a flare were to cool and give rise to the observed $\mathrm{H} \alpha$ emitting loops they would have to shrink in height. In another example, Forbes \& Acton (1996) associated the outer envelope of a cusped flaring soft X-ray emitting arcade with its inner envelope at a later time and interpreted the difference in heights as due to the shrinkage of coronal loops.

* Movies are only available in electronic form at http://www.edpsciences.org
More compelling evidence for coronal loop retraction during solar flares has been based on the interpretation of soft X-ray observations of supra-arcade downflows (McKenzie \& Hudson 1999, 2001; McKenzie 2000). These downflows are dark bloblike features which propagate sunward with speeds in the range $\sim 35-500 \mathrm{~km} \mathrm{~s}^{-1}$. They occur between soft X-ray emitting rays located above a bright flaring arcade of closed loops; a structure first reported by Švestka et al. (1998) and referred to as a fan of coronal rays. The dark supra-arcade downflows were observed clearly during the decay phase of flares, and appear to be best seen in intense long duration events (LDEs). They were interpreted by McKenzie \& Hudson $(1999,2001)$ and McKenzie (2000) as due to newly reconnected magnetic loops seen "edgeon" (i.e., where major axis of the loop is in a plane nearly parallel to the line of sight) retracting sunward.

Similar supra-arcade downflows have now been observed in EUV observations from the Transition Region and Coronal Explorer (TRACE) (Handy et al. 1999) for events on 2002 April 21 (Gallagher et al. 2002; Innes et al. 2003a,b; Sheeley \& Wang 2002; Sheeley et al. 2004), 2002 July 23 (Asai et al. 2004), and 2003 November 04 (Sheeley et al. 2004). Asai et al. (2004) showed that the supra-arcade downflows for the particular event they examined occurred during the rise phase of the flare and appeared to correlate well with the times of hard $\mathrm{X}$-ray bursts. This supports the suggestion that the downflows are associated with the snapping back of magnetic field lines during the magnetic reconnection process (or equivalently, magnetic reconnection outflow jets). In TRACE EUV observations the downflows appear as sunward propagating dark regions with trailing dark regions with a tadpole-like or comet-like appearance. Recently, Sheeley et al. (2004) reported observations of dark loop-shaped downflows. This appears to support the suggestion that dark supra-arcade downflows are due to shrinking loops. It should be noted that supra-arcade downflows generally 


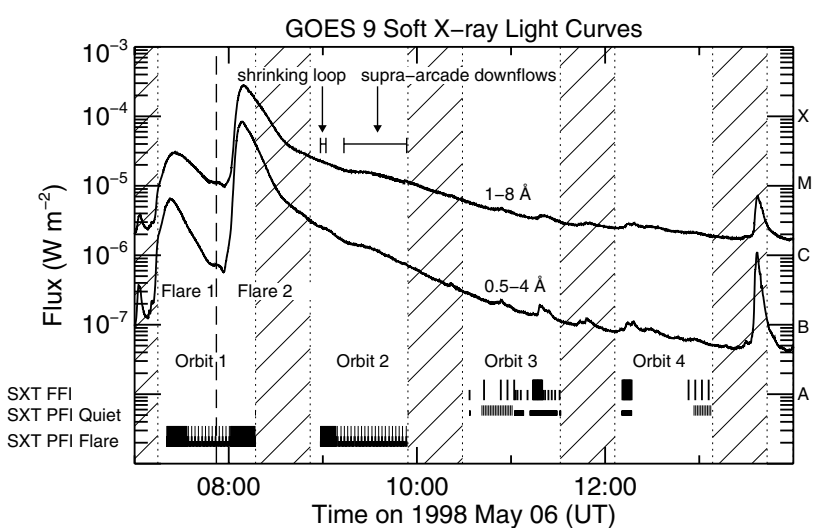

Fig. 1. The GOES 9 soft X-ray fluxes showing the times of SXT fullframe images (FFIs) and quiet-mode and flare-mode partial-frame images (PFIs). Also shown are the intervals of the enumerated flares, shrinking loop, supra-arcade downflows and Yohkoh satellite night (hatched intervals).

appear dark in soft X-ray and EUV images. Spectroscopic studies of supra-arcade downflows by Innes et al. (2003a), which show that they are dark in lines sensitive to plasmas over the temperature range $10^{4}-10^{7} \mathrm{~K}$, indicate that they are due to voids of plasma, rather than cooler absorbing plasma.

While the supra-arcade downflows are generally dark, bright soft X-ray emitting supra-arcade downflows have also been observed (McKenzie 2000). Moreover, McKenzie (2000) also reported distinct soft X-ray emitting loops which were observed to shrink into a bright flaring arcade.

Images from the Large Angle Spectroscopic Coronagraph (LASCO) (Brueckner et al. 1995) on board the Solar and Heliospheric Observatory (SOHO) (Domingo et al. 1995) have also revealed similar sunward dark (loop-shaped) downflows (i.e., voids in emission) (Wang et al. 1999; Sheeley \& Wang 2001; Sheeley et al. 2001, 2004).

In this paper we present what we believe is the first report of direct observations of simultaneous retracting and expanding distinct coronal loops observed in emission in soft X-rays during a solar flare. Both loops were observed nearly face-on (i.e., with the loop major axis approximately orthogonal to the line of sight). An analysis of observations relating to the retracting and expanding coronal loops is presented in Sect. 2. A discussion of our results and conclusions are presented in Sect. 3. We also present movies of the shrinking and expanding coronal loops in Appendix A.

\section{Observations}

\subsection{Whole-Sun soft $X$-ray fluxes}

Figure 1 shows broadband, whole-Sun soft X-ray fluxes from detectors on board the Geostationary Operational Environmental Satellite (GOES) 9 for times near the shrinking coronal loop. The detectors, covering the wavelength ranges $\sim 0.5-4 \AA$ and 1-8 $\AA$, are used here to examine the soft X-ray flare associated with the shrinking soft $\mathrm{X}$-ray loop and the temporal context in which the shrinking loop occurred. On the plot we use hatched intervals to indicate when Yohkoh was behind the Earth (and did not observe the Sun) and tick marks (which sometimes appear as bands) to indicate the times of imaging data examined in our analysis.

In Fig. 1 we also indicate the time intervals when the bright shrinking coronal loop and the dark supra-arcade downflows

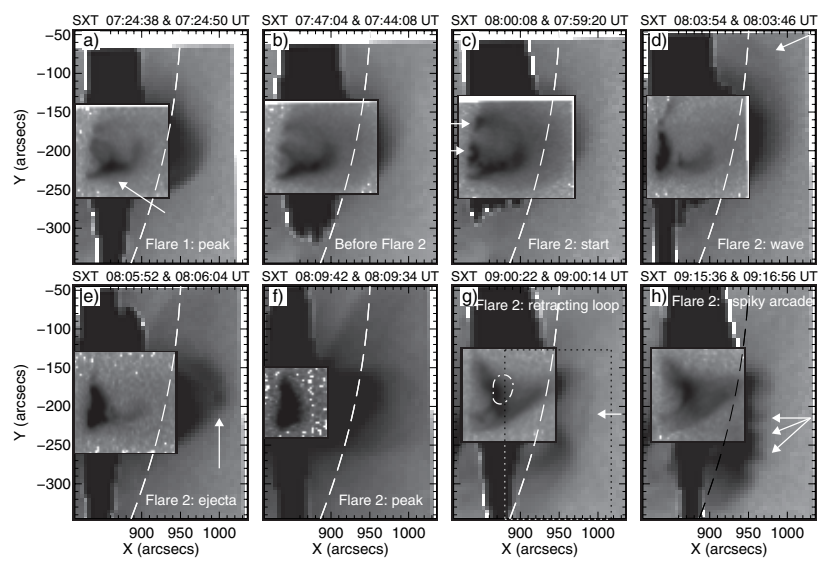

Fig. 2. Portions of corrected SXT PFIs showing an overview of the development of the sequence of Flares 1 and 2 on 1998 May 06. The images have been scaled to show the brightest features (using a reverse black and white colour table where dark denotes high intensity) in each image (relative differences between images are not retained). Each panel consists of a half-resolution (4.91" pixel) image with a superposed full-resolution $\left(2.45^{\prime \prime}\right.$ pixel) image with the times for each, respectively, indicated above each panel. The white dashed curves indicate the solar limb. The white arrows indicate features mentioned in the main text. The white (dot-dash) contour in (g) indicates the HXT L channel $(\sim 14-23 \mathrm{keV})$ source observed during the time of the shrinking loop.

were observed in the SXT data. The shrinking loop occurred during the decay phase of an X2.7 solar flare which occurred from 07:52-13:22 UT on 1998 May 06 at $\sim$ S15W66 in NOAA Active Region 8210. The time of peak (GOES) soft X-ray flux for the flare was 08:09 UT. This flare was preceded by an M2.9 flare which occurred from $\sim 07: 10-07: 52$ UT at the nearby location of $\sim \mathrm{S} 16 \mathrm{~W} 70$. Here we refer to the M-class flare as "Flare 1" and the X-class flare as "Flare 2". Some aspects of Flare 2 and phenomena associated with it have already been examined and reported elsewhere (e.g., Khan \& Hudson 2000; Klassen et al. 2001; Hudson et al. 2003). In this paper we concentrate on aspects related to the shrinking and expanding coronal loops associated with Flare 2.

\subsection{Yohkoh soft $X$-ray telescope images}

We examined whole-Sun full-frame images (FFIs) as well as quiet-mode and flare-mode partial-frame images (PFIs) from SXT for Orbits 1-4 as indicated in Fig. 1. An overview of the development of Flares 1-2 for Orbits 1-2 as seen in SXT PFIs is shown in Fig. 2. In each panel of this figure we present a halfresolution (4.91" pixel) image to show the faint outer structures with a superposed full-resolution $\left(2.45^{\prime \prime}\right.$ pixel) image to show the brighter flaring inner structures.

Figure 2a shows images of Flare 1 for a time near its peak $(G O E S)$ soft X-ray flux. Comparisons with images of Flare 2 seen later indicate that Flare 1 originated from loops near to those seen for Flare 2. We find no evidence in the SXT data to indicate that Flare 1 was associated with dynamic phenomena such as ejecta, in contrast with Flare 2. Figure $2 b$ shows images of Flare 1 during its decay phase, just before Flare 2 started. The remaining images in Fig. 2 relate to Flare 2.

Near the start of Flare 2 a loop with bright footpoints and a smaller remote loop (apparently connected to it) were observed (these two small loops are indicated by white arrows in Fig. 2c. See also Fig. 3 in Hudson et al. 2003). Later a wave-like signature, inferred to be the direct observation of a shock wave 

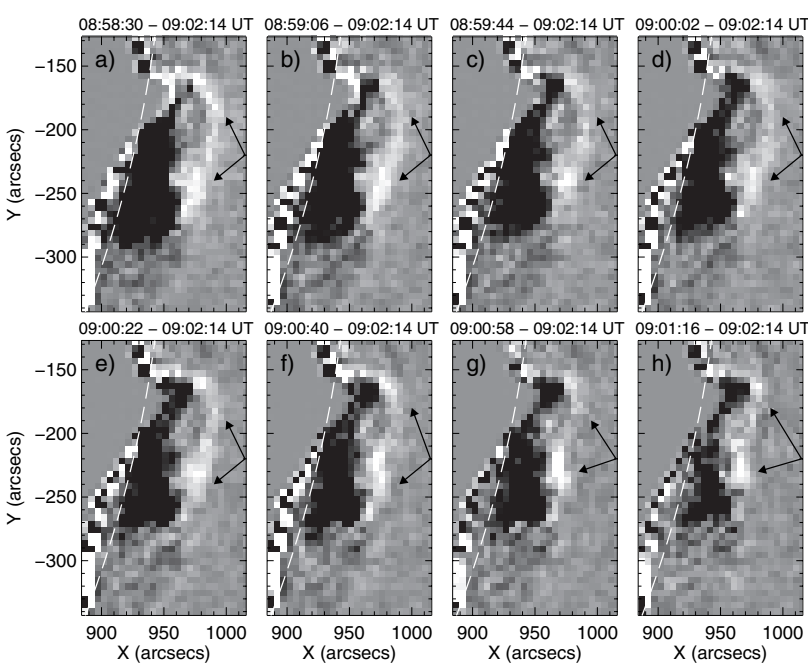

Fig. 3. Portions of difference images obtained by subtracting the corrected SXT half-resolution (4.91" pixel) PFI taken at 09:02:14 UT from other PFIs. The white dashed curve indicates the solar limb. The white (and hence bright) feature indicated by the black arrows is the shrinking coronal loop which is observed in emission in soft X-rays.

seen in soft X-rays (Khan \& Hudson 2000; Hudson et al. 2003) was observed (indicated by the white arrow in Fig. 2d) to emanate from the vicinity of Flare 2. After this, ejecta were observed above the bright flaring loop (indicated by white arrow in Fig. 2e). Near the time of peak soft X-ray emission, Flare 2 appeared to be a bright loop or set of loops (Fig. 2f). We refer to the region of brightest flaring emission observed in Figs. $2 d-f$ as the flare kernel.

At the start of Orbit 2, from 08:58:30-09:01:58 UT, a loop observed faintly in emission (indicated by the white arrow in Fig. 2g) was seen to retract into the region between the bright arcade above the flare kernel and a bright set of loops which appeared to the south. After the loop retracted, from $\sim 09: 13: 28-09: 53: 28 \mathrm{UT}$, the underlying loops appeared to show dynamic behaviour exhibiting the fan of coronal rays phenomenon with dark supra-arcade downflows (indicated by white arrows in Fig. 2h).

For Orbits 3-4 the PFIs show the flare kernel but no remarkable dynamic phenomena, while the FFIs show the fan of coronal rays phenomenon. The time cadence of the FFIs is inadequate to observe downflows. However, the changed positions of the rays in the images is suggestive of the apparent wiggling of rays observed in other events. Supra-arcade downflows may still have been occurring during those Yohkoh orbits.

The development of the shrinking loop associated with Flare 2 is shown in detail in Fig. 3 as a series of difference images. For each (half-resolution) SXT image, we subtracted the image taken at $~ 09: 02: 14 \mathrm{UT}$, when the shrinking loop was no longer visible. The SXT images, as well as the difference images, show a retracting distinct loop structure observed faintly in emission located above the brighter loop structures. The retracting loop is seen more clearly in movies of these images.

To determine the speed of the retracting coronal loop we used the following procedure. First consider a single image. We estimated the location of the loop by determining the brightest pixels along the loop. These and adjacent pixels were used to define the "ridge" or arc of peak values along the loop. An example of an estimated loop arc is shown in black in Fig. 4a. We then treated this arc or line of pixels as a template for the loop, or as an accumulator, as is used in e.g., a generalised Hough transform analysis, although we do not carry out such a detailed analysis
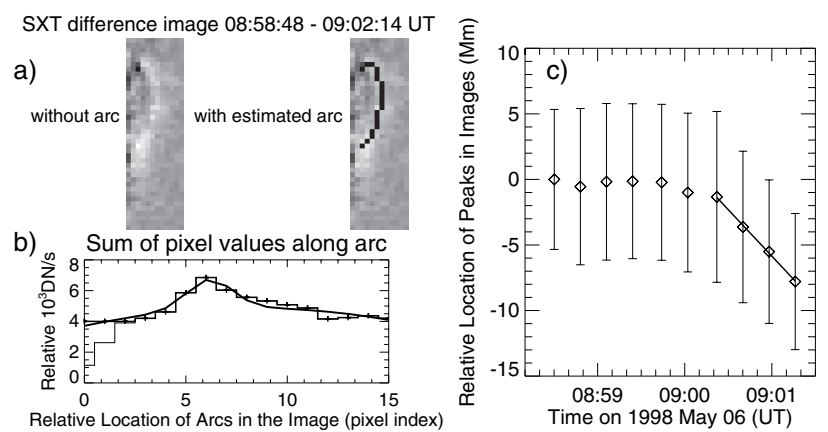

Fig. 4. This figure indicates the technique used to estimate the speed of the shrinking coronal loop. a) The first panel is part of a difference image showing the (shrinking) coronal loop. The second panel shows the same image with, superposed, an arc of black pixels indicating the estimated loop position (i.e., a template for the loop). b) The loop template is shifted horizontally across the image and the sum of intensity values of the pixels lying within the template are plotted versus the pixel shift position. Low intensity values sunward of the intensity peak were set to values close to the lowest values on the other side of the peak. The peak in plot b) is used to determine the loop location, while the full-width at half-maximum ( $F W H M$ ) of the intensities is used as an estimate of the uncertainty in location. c) A plot of intensity peak locations versus time determined from a series of difference images. A least-squares linear fit to the last four points with error bars corresponding to the FWHM of the intensities indicates that the speed of the loop was $118 \pm 144 \mathrm{~km} \mathrm{~s}^{-1}$. However, assuming a single pixel uncertainty over the linearly increasing separation interval leads to a speed estimate of $118 \pm 66 \mathrm{~km} \mathrm{~s}^{-1}$.

here. Instead, we simply shifted the line (i.e., loop template or accumulator) in the horizontal CCD direction in one pixel steps and for each location (with an assigned index value) we add the intensities of the pixels now within the template (i.e., along the accumulator). We then plot the accumulated intensities along the shifted arcs with horizontal location or index. An example of such a plot is shown in Fig. 4b. A few caveats should be noted. We do not use the raw images themselves as the bright emitting regions sunward of the shrinking loop complicate the analysis. Instead we use difference images like those shown in Fig. 3, but we adjust the values to have only positive values. Furthermore, as the differencing leads to rather low intensity values sunward of the shrinking loop we set the lowest summed values to values close to the lowest values on the other side of the intensity peak. This can also be seen in Fig. 4b. From this plot we fit a Gaussian plus quadratic to the data and estimate the (relative) location of the loop as the location of the peak in the Gaussian and the uncertainty (error bars) in location as the full-width at half-maximum $(F W H M)$ of the Gaussian. We repeat this procedure for each image. It should be noted that we define a different accumulator for each image. We then plot the loop locations for each image versus the time of each image. This plot is shown in Fig. 4c. A least-squares linear fit to the last four points with $F W H M$ error bars results in a speed estimate of $118 \pm 144 \mathrm{~km} \mathrm{~s}^{-1}$. However, the uncertainties indicated by the $F W H M$ error bars are clearly an over-estimate of the errors. If, instead, we assume a single pixel uncertainty over the linearly increasing separation interval we conclude that the speed of retraction of the loop was $118 \pm$ $66 \mathrm{~km} \mathrm{~s}^{-1}$.

We also draw attention to another interesting feature in the SXT data. In quarter resolution (9.82" pixel) PFIs, as well as observing the shrinking loop, the fan of coronal rays and dark supra-arcade downflows (all with coarser spatial resolution) we also observed an outward moving loop-like feature (observed faintly in emission) located $\sim 200^{\prime \prime}$ above the shrinking loop during the time of the shrinking loop. Figure 5 presents a series 

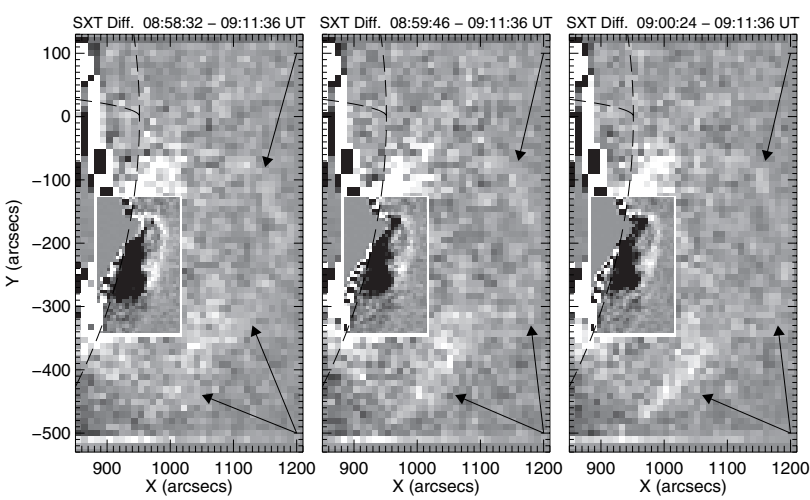

Fig. 5. Portions of difference images obtained by subtracting the corrected SXT quarter-resolution (9.82" pixel) PFI taken at 09:11:36 UT from other PFIs. We refer to the bright feature indicated by the arrows as the expanding coronal loop. Superposed on each image is the closest image in time from Fig. 3 which is used to indicate the shrinking coronal loop.

of difference images showing the outward moving loop. Using the same technique as that for the shrinking loop we estimate the speed of the outward moving loop was $\sim 129 \pm 74 \mathrm{~km} \mathrm{~s}^{-1}$. The outward moving loop is seen more clearly in movies of these images.

\subsection{Hard X-rays}

We examined hard X-rays observed during Flare 2 in data from (i) the Discriminator Large Area (DISCLA) detectors on the Burst and Transient Source Experiment (BATSE) on board the Compton Gamma-ray Observatory (Fishman et al. 1989) and (ii) the Yohkoh Hard X-ray Telescope (HXT) (Kosugi et al. 1991). Figure 6 shows the BATSE DISCLA data in the energy bands $\sim 25-50 \mathrm{keV}$ and $\sim 50-100 \mathrm{keV}$ for times near the start of Flare 2 until after the shrinking coronal loop. Inset in this figure we show a zoomed portion of this BATSE data together with HXT L channel ( 14-23 keV), M1 channel ( 23-33 keV), and M2 channel ( $\sim 33-53 \mathrm{keV})$ data for times near the shrinking coronal loop. These data sets indicate that small hard $\mathrm{X}$-ray bursts occurred in the BATSE DISCLA $\sim 25-50 \mathrm{keV}$ and $\sim 50-100 \mathrm{keV}$ energy bands as well as in the HXT L, M1, and M2 channel data during the interval of coronal loop shrinkage. We are able to construct several images of the HXT L channel data for times overlapping the interval of the shrinking loop. A representative example is shown as the white (dot-dash) contour in Fig. 2g. We find that the (HXT L channel) hard X-ray emission originated from the flare kernel. Due to the relatively low counts in the HXT M1 and M2 channel data during the interval of the shrinking loop we are unable to construct images for those channels.

\section{Discussion and conclusions}

In this Paper we have presented images from Yohkoh SXT which appear to indicate the retraction of a distinct coronal loop as well as an outward moving coronal loop, both observed in emission in soft X-rays during the decay phase of a solar flare. We estimate the speeds of the retracting coronal loop and outward moving coronal loop were $118 \pm 66 \mathrm{~km} \mathrm{~s}^{-1}$ and $\sim 129 \pm 74 \mathrm{~km} \mathrm{~s}^{-1}$, respectively. The speeds we find for the shrinking loop are similar to the reported speeds of dark SXT supra-arcade downflows (McKenzie \& Hudson 1999; McKenzie 2000). McKenzie \& Hudson (2001) have suggested that such speeds are not

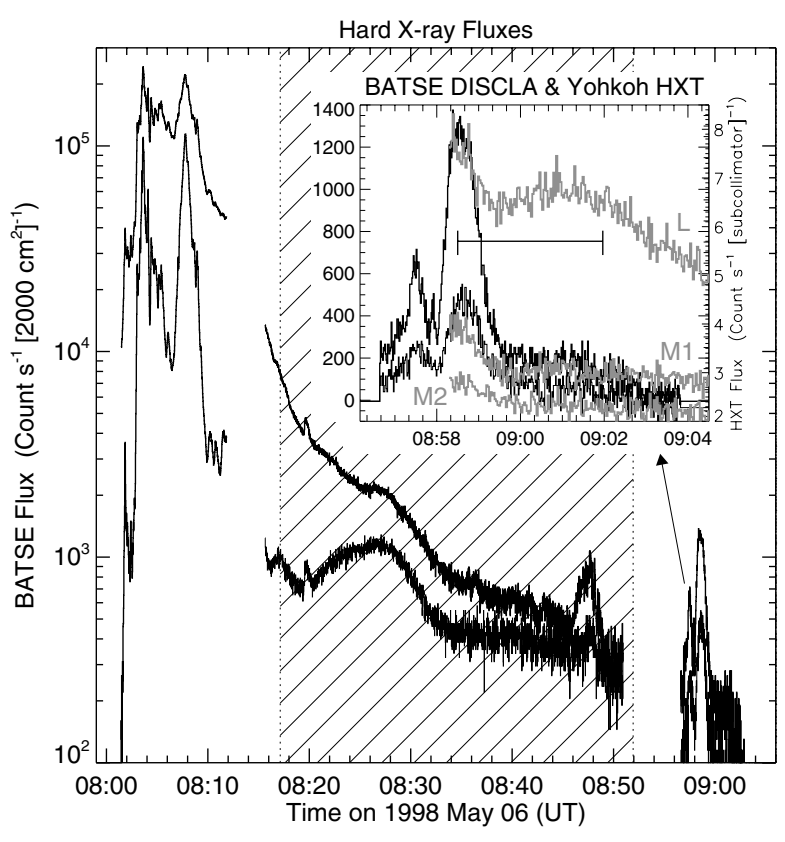

Fig. 6. Hard-ray fluxes from the BATSE DISCLA $\sim 25-50$ and $\sim 50-100 \mathrm{keV}$ channel data for Flare 2. The hatched interval indicates Yohkoh satellite night. Inset shows the BATSE DISCLA data and HXT L, M1 and M2 channel data near the time of the shrinking coronal loop (interval indicated).

necessarily inconsistent with reconnection outflow speeds, which may be significantly lower than the Alfvén speed in the outflow region. A reconnection outflow emerging into a compressible, post-reconnection region with plasma density larger than that of the inflow region does so at speed less than the Alfvén speed at the inflow region (Priest \& Forbes 2000), by a factor of $\left(\rho_{i} / \rho_{o}\right)^{1 / 2}$, where $\rho$ denotes the plasma mass density and the subscripts $i, o$ denote the inflow and outflow regions. (Note, this is based on mass conservation in a Sweet-Parker sheet configuration - the simplest case). Thus a mass (and hence particle number) density ratio of a factor 0.01 between the inflow and outflow regions would be consistent with an outflow speed a factor of ten less than the typical coronal Alfvén speed (at the inflow region). Assuming an Alfvén speed (at the inflow region) of $\sim 2000 \mathrm{~km} \mathrm{~s}^{-1}$ (due to a particle number density, $n \sim 10^{8} \mathrm{~cm}^{-3}$ and a magnetic field strength, $B \sim 10 \mathrm{G}$ ) requires a number density in the post-reconnection region of $\sim 10^{10} \mathrm{~cm}^{-3}$. This may be reasonable once the flare has entered its gradual phase, when the shrinking loop is seen.

As noted by Hudson \& McKenzie (2001) if supra-arcade downflows indicate recently reconnected magnetic field lines then both upward and downward flows might be expected. Upward dark supra-arcade flows have not been reported in the imaging SXT or TRACE data. However, Asai et al. (2004) suggest that soft X-ray emitting, so-called plasmoid ejections (e.g., Ohyama \& Shibata 1997, 1998; Khan et al. 2002) may be the upflow counterpart of dark supra-arcade downflows. However, it is not clear why the downflows are dark while the upflows are bright. Note, for the event examined here, we find a shrinking loop and simultaneous outward moving loop both observed in emission. These may indicate the snapping of magnetic field lines both downwards and upwards, respectively, during the magnetic reconnection process. In other words, these observations may provide further evidence for reconnected magnetic field lines during a flare.

As the retracting loop reported here was observed in emission in soft X-rays, it clearly was not a magnetic flux tube 
essentially void of plasma, as has been found for dark supraarcade downflows (Innes et al. 2003a). The magnetic flux tube was loaded with mass which must have had a temperature $\gtrsim 2 \mathrm{MK}$ to be visible with the SXT. The same applies to the simultaneous outward moving loop. If we assume a temperature in the range $\sim 3-10 \mathrm{MK}$ and a line-of-sight thickness of $10^{4} \mathrm{~km}$, the SXT data numbers $(\mathrm{DN})$ per second per pixel $(\sim 800$ for the AlMg. filter) for the shrinking loop and a vignetting correction of 0.79 imply a particle number density of $\sim 10^{10} \mathrm{~cm}^{-3}$ consistent with the speed estimates given earlier.

If the shrinking loop is evidence for reconnected magnetic field lines a direct association with hard X-ray bursts might be expected. While the temporal overlap of the observed hard $\mathrm{X}$-ray bursts up to $\sim 100 \mathrm{keV}$ appear to support this suggestion, the source locations of the $\sim 14-23 \mathrm{keV}$ hard X-ray data do not. Although the location of the small hard X-ray bursts is not near the shrinking or expanding loops this does not necessarily rule out an association between the hard X-rays and the moving loops. Since hard X-rays are due to accelerated particles, they can be located far from the reconnected soft X-ray structures so long as they are, or were, magnetically connected to them. It is also possible that the lower energy hard X-rays were dominated by thermal emission from the flare kernel, but higher energy hard X-rays may have been associated with the shrinking loop. However, as we are unable to construct images of the higher energy hard X-rays we are unable to confirm or refute this possibility.

The shrinking loop was observed from $~ 08: 58: 30$ 09:01:58 UT. Then from $\sim 09: 13: 28-09: 53: 28$ UT the loops of the arcade into which the loop retracted showed dynamic behaviour exhibiting the fan of coronal rays phenomena and dark supra-arcade downflows. During the latter interval, the soft $\mathrm{X}$-ray emission from the southern arcade and the supra-arcade region above it increased significantly. We note a change in slope of the GOES soft X-ray flux during the time of the supra-arcade downflows (see Fig. 1). Concerning this spatial and temporal development: We would like to ascertain if the shrinking loop caused (e.g., by reconnecting with the underlying arcade) the fan of coronal rays (between which the dark supra-arcade downflows were seen), or if it indicates an association of the shrinking loop and the supra-arcade downflows with some other process (e.g., both represent retracting loops from the reconnection region), or if it was merely coincidental. Unfortunately, the data for this event do not allow us to discriminate between these possibilities.

We carried out a survey of all half-resolution flare-mode SXT PFIs for M- and X-class solar flares to search for other shrinking loop events observed in emission. While we find a couple of possibilities, none are as convincing or clear as this event. We speculate that the M-class flare, Flare 1, may have increased the temperature and density of its surroundings allowing us to see the relaxing magnetic structures of the subsequent $\mathrm{X}$-class flare, Flare 2, in emission in soft X-rays. More specifically, perhaps having two large flares in close succession, but where the first flare is relatively confined is an important factor. As Flare 1 was a relatively confined flare, hot flaring plasma might have been contained in the coronal loop structures which eventually reconnected during Flare 2.

We also note that the bright shrinking loop and simultaneous outward moving loop were observed nearly face-on in our event, but shortly afterwards dark supra-arcade downflows were seen, which have been interpreted as edge-on loops. That is, there appear to have been roughly orthogonal retracting magnetic loops in this event. We can think of two possibilities to explain these observations. The bright shrinking and outward moving loops may be related to the magnetic field of a prominence field located within the arcade which could have had a significant component orthogonal to the overlying arcade field (which may relate to the later supra-arcade downflows). Alternatively, the flaring arcade could have an elongated and twisted shape where face-on and edge-on loops occur nearly along the line-of sight for this near-limb event. In that case, the loops for the face-on part of the arcade may have retracted initially, followed by the loops for the edge-on part.

We anticipate significant advances in our understanding of the dynamics (inflows/outflows and loop shrinkage/expansion) relating to the magnetic reconnection process in solar flares to be made with new data from the Solar-B Mission. Solar-B, a mission of the Institute of Space and Astronautical Science (ISAS) of Japan built in collaboration with US and UK scientists will provide observational data particularly useful in the study of faint, flare-associated, outflow phenomena. Since the Solar-B $\mathrm{X}$-ray Telescope (XRT) will provide the highest spatial and temporal resolution soft X-ray images ever obtained and moreover over a wider temperature range and with low scattering it will provide much better soft X-ray images of such faint features, while the Solar-B EUV Imaging Spectrometer (EIS) will provide the means to study them spectroscopically. Thus it will be possible to determine the physical conditions near the flare energy release site and the detailed development of the magnetic field changes. These will help advance the understanding of the flare energy release process.

Acknowledgements. J.I.K. gratefully acknowledges financial support from the UK Particle Physics and Astronomy Research Council (PPARC).

\section{References}

Asai, A., Yokoyama, T., Shimojo, M., \& Shibata, K. 2004, ApJ, 605, L77 Brueckner, G. E., Howard, R. A., Koomen, M. J., et al. 1995, Sol. Phys., 162, 357

Domingo, V., Fleck, B., \& Poland, A. I. 1995, Sol. Phys., 162, 1

Fishman, G. J., Meegan, C. A., Wilson, R. B., et al. 1989, in Proc. GRO Science Workshop, ed. W. N. Johnson (Greenbelt: NASA), 2

Forbes, T. G., \& Acton, L. W. 1996, ApJ, 459, 330

Gallagher, P. T., Dennis, B. R., Krucker, S., Schwartz, R. A., \& Tolbert, K. 2002, Sol. Phys., 210, 341

Handy, B. N., Acton, L. W., Kankelborg, C. C., et al. 1999, Sol. Phys., 187, 229 Hirayama, T. 1974, Sol. Phys., 34, 323

Hudson, H. S. 2000, A\&A, 531, L75

Hudson, H. S., \& McKenzie, D. E. 2001, Earth Planets Space, 53, 581

Hudson, H. S., Khan, J. I., Lemen, J. R., et al. 2003, Sol. Phys., 212, 121

Innes, D. E., McKenzie, D. E., \& Wang, T. 2003a, Sol. Phys., 217, 247

Innes, D. E., McKenzie, D. E., \& Wang, T. 2003b, Sol. Phys., 217, 267

Khan, J. I., \& Hudson, H. S. 2000, Geophys. Res. Lett., 27, 1083

Khan, J. I., Vilmer, N., Saint-Hilaire, P., \& Benz, A. O. 2002, A\&A, 388, 363

Klassen, A., Aurass, H., \& Mann, G. 2001, A\&A, 370, L41

Kopp, R. A., \& Pneuman, G. W. 1976, Sol. Phys., 50, 85

Kosugi, T., Makishima, K., Murakami, T., et al. 1991, Sol. Phys., 136, 17

McKenzie, D. E. 2000, Sol. Phys., 195, 381

McKenzie, D. E., \& Hudson, H. S. 1999, ApJ, 519, L93

McKenzie, D. E., \& Hudson, H. S. 2001, Earth Planets Space, 53, 577

Ohyama, M., \& Shibata, K. 1997, PASJ, 49, 249

Ohyama, M., \& Shibata, K. 1998, ApJ, 499, 934

Priest, E. R., \& Forbes, T. 2000, Magnetic Reconnection: MHD Theory and Applications (Cambridge: Cambridge University Press)

Sheeley, N. R., Jr., \& Wang, Y.-M. 2001, ApJ, 562, L107

Sheeley, N. R., Jr., \& Wang, Y.-M. 2002, ApJ, 579, 874

Sheeley, N. R., Jr., Knudson, T. N., \& Wang, Y.-M. 2001, ApJ, 546, L131

Sheeley, N. R., Jr., Warren, H. P., \& Wang, Y.-M. 2004, ApJ, 616, 1224

Švestka, Z. F., Fontenla, J. M., Machado, M. E., Martin, S. F., \& Neidig, D. F. 1987, Sol. Phys., 108, 237

Švestka, Z., Fárník, F., Hudson, H. S., \& Hick, P. 1998, Sol. Phys., 182, 179

Tsuneta, S., Acton, L. W., Bruner, M. E., et al. 1991, Sol. Phys., 136, 37

Wang, J., Shibata, K., Nitta, N., et al. 1997, ApJ, 478, L41

Wang, Y.-M., Sheeley, N. R., Jr., Howard, R. A., St. Cyr, O. C., \& Simnett, G. M. 1999, Geophys. Res. Lett., 26, 1203 